scenarios to be modelled by the ABM - to facilitate discussion and solicit feedback on the conceptual model specifications. The feedback from the sessions were collated and reviewed and adjustments were made to both the conceptual model and the use-cases.

Results Upon reaching a shared understanding of the model concept, participants identified gaps in the conceptual model and developed new use case scenarios to be adjusted for future iterations. A number of feedback loops in the model were also highlighted for further consideration (e.g., travel choice, food price elasticity). Data collected from the session also supplied valuable input into development of suitable visual boundary objects that will be used to facilitate additional group modelling exercises and conversations with stakeholders. Additional meetings with stakeholders will further refine the conceptual model and provide ground truthing to the computational model.

Conclusion This iterative process facilitated the understanding of the complex systems underpinning local food environments and allows for ground truthing and future validation of the ABM. Co-development of model use cases facilitated a shared understanding of the purposes of the model and will help to maximise its usefulness for stakeholders.

\section{P26 HOW DOES CHANGING THE PLACEMENT OF FOOD PRODUCTS IN SUPERMARKETS INFLUENCE CUSTOMERS' PURCHASING AND STORE SALES?}

${ }^{1} \mathrm{~S}$ Crozier*, ${ }^{1,2} \mathrm{~J}$ Baird, ${ }^{1,2} \mathrm{C}$ Cooper, ${ }^{1,2} \mathrm{C}$ Vogel. ${ }^{1} \mathrm{MRC}$ Lifecourse Epidemiology Unit, University of Southampton, Southampton, UK; ${ }^{2}$ NIHR Southampton Biomedical Research Centre, University Hospital Southampton NHS Foundation Trust, Southampton, UK

\subsection{6/jech-2020-SSMabstracts. 122}

Background Supermarkets are a major source of food for families, yet greater understanding of how product placement strategies influence the healthfulness of food choice is needed. We assessed the effect of improving the availability and positioning of fruit and vegetables in supermarkets, and removing confectionery from checkouts, on household-level purchases and store-level sales.

Methods This study was a natural experiment with a prospective matched cluster design set in a discount supermarket chain in England. The intervention had two components: new fresh fruit and vegetable sections at store entrances (replacing smaller displays at the back), and removal of confectionery from checkouts (replacing with healthier items, such as sugarfree gum, water and non-food items) and end-of-aisle opposite checkouts. Women customers aged 18 to 45 years, with a store loyalty card, who regularly shopped at three intervention and three matched control stores in England were recruited. Data from purchases using loyalty cards and for store sales were collected for the three months before and six months after refurbishment. Individual purchasing data were analysed using a difference-in-difference method, while store sales data were analysed using controlled interrupted time series by store pair, with differences synthesised using random-effects metaanalysis.

Results A total of 107 women provided household-level purchasing data. The proportion purchasing fresh fruit and vegetables per week rose in intervention stores at three months $(0.2 \%)$ compared to a drop in control stores $(-3.0 \%)$ $(\mathrm{P}=0.22)$, and at six months $(1.7 \%$ vs $-3.5 \%, \mathrm{P}=0.05)$. The proportion purchasing healthier checkout items rose more in intervention stores compared to a drop in control stores at both three $(1.0 \%$ vs $-1.8 \%, \mathrm{P}=0.04)$ and six $(0.6 \%$ vs $-1.6 \%$, $\mathrm{P}=0.13)$ months. There were no differences in the purchases of frozen fruit and vegetables or confectionery. Increases in store-level sales of fresh fruit and vegetables were greater in intervention stores than predicted at three months (1.71SDs (95\% CI 0.45, 2.96), $\mathrm{P}=0.01$ ) and six months (2.42SDs $(0.22$, 4.62), $\mathrm{P}=0.03)$. Decreases in sales of confectionery were greater in intervention stores than predicted at three months (-1.05SDs $(-1.98,-0.12), \mathrm{P}=0.03)$ and six months $(-1.37$ SDs ($2.95,0.22), P=0.09)$. Sales of frozen fruit and vegetables and healthier checkout items showed no differences.

Conclusion This study provides some evidence to suggest that healthier product placement in supermarkets improves the healthfulness of both household-level purchasing and storelevel sales. Improving fruit and vegetable placement should be considered by government alongside current plans to limit prominent placement of unhealthy foods.

\section{P27 DIET - IS THERE A NEW DIGITAL DIVIDE? SOCIAL INEQUALITIES IN USE OF DIGITAL FOOD DELIVERY SERVICES AND ASSOCIATIONS WITH BMI}

${ }^{1} \mathrm{~S}$ Cummins ${ }^{*},{ }^{1} \mathrm{~N}$ Berger, ${ }^{1} \mathrm{~V}$ Er, ${ }^{2} \mathrm{C}$ Thompson, ${ }^{1} \mathrm{C}$ Law, ${ }^{1} \mathrm{~L}$ Cornelsen. ${ }^{1}$ Population Health Innovation Lab, London School of Hygiene and Tropical Medicine, London, UK; ${ }^{2}$ School of Health and Social Work, University of Hertfordshire, Hatfield, UK

10.1136/jech-2020-SSMabstracts. 123

Background Food retailing is undergoing a fundamental transformation. Digital on-demand technology is dramatically reshaping food distribution and delivery, making food and prepared meals more accessible and convenient. The impact of this disruption on food purchasing and consumption behaviour and dietary inequalities is unknown. This study aims to explore whether there is a social gradient in use of digital food delivery services for both take-away food and grocery purchasing, and whether use of these services is associated with BMI.

Methods We used data from UK Kantar Fast Moving Consumer Goods (FMCG) panel, a nationally representative panel study of all take-home household food and beverage purchasing. We used data from London and the North West of England $(n=1559)$ from February 2019. Households reported usage of digital take-away food delivery services (past 7 days) through a bespoke online survey. We used logistic regression models to estimate the odds of delivery service usage by occupational grade $(\mathrm{AB}, \mathrm{C} 1, \mathrm{C} 2, \mathrm{DE})$ and income $(£ 0-20 \mathrm{~K}, £ 20+-$ $50 \mathrm{~K}, £ 50 \mathrm{~K}+$ ) and to estimate whether usage was associated with BMI category $(<25 ; 25-29 ;>30)$, derived from selfreported height and weight. Results were adjusted for potential socio-demographic confounders.

Results For take-away food purchasing, 13\% of survey respondents had used a digital delivery service in the past 7 days. In fully adjusted models, a dose-response pattern was observed with lower occupational grade associated with higher usage for both C2 (OR 1.66, 95\% CI 1.01,2.73) and DE (OR 1.92, 95\% CI 1.18,3.09) households, compared to AB. Similar patterns were observed for household income. Use was associated with BMI category 25-29 (OR 1.52, 95\% CI 1.10, $2.29)$ and $30+(\mathrm{OR} 1.8,95 \%$ CI 1.18, 2.72), compared to $<25$. For digital grocery purchasing, $15.6 \%$ of households had 